\title{
Das Unmögliche möglich machen
}

_ Halten Sie den Direct Access für genauso unwahrscheinlich wie die Marsmission? Ich nicht! Noch weit vor 2037 wird es Patienten erlaubt sein, mit Beschwerden am Bewegungssystem direkt einen Physiotherapeuten aufsuchen zu können, ohne den Umweg über den Arzt gehen zu müssen. Davon bin ich überzeugt. Warum? Keiner kann bei Störungen im und Schmerzen am Bewegungssystem so gut einen klinischen Befund erheben und verfügt über so profunde manuelle Fertigkeiten wie die Physiotherapeuten (๑ S. 8).

_ Qualität setzt sich auf Dauer durch. Ob allerdings für den Direct Access ein Studium nötig ist, bezweifle ich sehr. Warum sollte ein Physiotherapeut mit fundierten Kenntnissen in manueller Therapie weniger kompetent für den Direktzugang sein als beispielsweise ein Physiotherapeut mit Bachelorabschluss? Unabhängig davon ist der Akademisierungsprozess natürlich trotzdem ein Muss!

_ Vor den Kürzungsplänen der Regierung Obamas hatte die NASA für 2037 den ersten bemannten Flug zum Mars anvisiert. Ein unglaubliches Unterfangen. Alleine für den Hinflug zum Mars wären die Astronauten abhängig von der Flugbahn circa 500 Tage unterwegs. Der Langzeitrekord bemannter Raumflüge liegt derzeit bei 437 Tagen. Die Anziehungskraft des Mars beträgt nur ein Drittel der Erdanziehung. Immerhin, denn dadurch würde sie der Besatzung eine gewisse Regeneration der Muskulatur und Knochen ermöglichen. In der Schwerelosigkeit kommt es trotz intensiven Trainings $z u$ Muskelatrophie und Osteoporose. Deshalb spielt die Physiotherapie in der Raumfahrt ein wichtige Rolle ( $\odot$ S. 38). Die Idee einer Marsmission wurde in den 1960er-Jahren geboren, im Rahmen der ersten bemannten Landung auf dem Mond. Es sind noch einige technische Hürden zu überspringen, und es werden sicher noch etliche Jahre vergehen müssen, bis sie wahr werden wird.

_ Bei den Psychotherapeuten hat es etwa 20 Jahre gedauert, bis Patienten mit ihrem Versichertenkärtchen direkt zur Psychotherapie gehen konnten. Wir müssen uns also etwas gedulden, bis das für die Ärzte scheinbar Unmögliche möglich wird - der Direktzugang zum Physiotherapeuten.

Herzlich Ihre

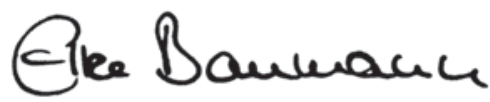

\section{PHYSIOBONUS}

physiobonus heißen die Gewinnspiele in physiopraxis. Wer teilnehmen möchte, nutzt das Formular unter www.thieme.de/ physioonline in der Rubrik physioexklusiv. Und das gibt es in dieser Ausgabe zu gewinnen:

Kursplatz

1 Kursplatz „Faszien- und Membrantechnik“ Seite 56

Bücher

2-mal „Teilhaben!“ $\quad$ Seite 30

3-mal „Prävention“ Seite 35

3-mal „Querschnitt“ Seite 36

1-mal „Faszien- und

Membrantechnik"

Seite 56

und außerdem

1 Tag im European

Astronaut Centre

Seite 40 